\title{
Die Soncino-Gesellschaft in der Presse ihrer Zeit
}

Die Zusammenstellung basiert auf der Pressemappe der Soncino-Gesellschaft im Nachlass von Herrmann Meyer im Archiv des Jüdischen Museums Berlin. ${ }^{1}$ Meyer hatte die Zeitungsausschnitte in einem dunkelroten Folioband gesammelt. Insgesamt finden sich hier beklebt 42 Seiten, mit 130 nummerierten Artikeln. Darüber hinaus gibt es Ausschnitte ohne Nummerierung sowie lose eingelegte Ausschnitte. Von Herrmann Meyer separat aufgeführt wurden Presseausschnitte zur Jahrestagung in Frankfurt am Main 1931.

\section{Artikel von 1 bis 130 in der Pressemappe nummeriert}

1) Jüdische Telegraphen-Agentur GmbH (Berlin), Jg. 3, Nr. 437 (21.06.1924) „Eine Gesellschaft der Freunde des jüdischen Buches“ [Gründungsbekanntgabe und Nennung der Mitglieder des Ehrenausschusses]

2) Frankfurter Zeitung (Frankfurt a. M.), Lokalteil „Stadtblatt“ (05.07.1924) „Die SoncinoGesellschaft“ [Gründungsbekanntgabe]

3) Wiener Morgenzeitung (Wien), Literaturbeilage „Das Buch der Zeit“, Jg. 6, Nr. 1925 (25.06.1924) „Aus der Werkstatt des Buches. Eine Gesellschaft der Freunde des jüdischen Buches" [Gründungsbekanntgabe]

4) Berliner Tageblatt und Handels-Zeitung (Berlin), Abend-Ausgabe, Jg. 53, Nr. 294 (24.06.1924) „Die Soncino-Gesellschaft“ [Gründungsbekanntgabe]

5) Jüdisches Wochenblatt (Berlin), Jg. 1, Nr. 16 (26.06.1924), S. 149 [Gründungsbekanntgabe, Nennung der Mitglieder des Ehrenausschusses]

6) Zionistische Korrespondenz (Berlin), Jg. 4, Nr. 25 (24.06.1924), S. 8, „Eine Gesellschaft der Freunde des jüdischen Buches“, Verfasser: Ziko (Zionistische Korrespondenz) [Gründungsbekanntgabe der Soncino-Gesellschaft]

7) Jüdische Rundschau (Berlin), Jg. 29, Nr. 51 (27.06.1924), S. 367, „Die SoncinoGesellschaft“, Verfasser: Arnold Zweig [Artikel zu Gründung und Vorhaben der Gesellschaft]

8) Vossische Zeitung: Berlinische Zeitung von Staats- und gelehrten Sachen (Berlin), Morgenausgabe, Nr. 302 (27.06.1924), Neuer Tagesdienst der Vossischen Zeitung, Ergänzung zur Berliner Ausgabe, [S. 11] „Eine neue Bibliophilen-Gesellschaft“ [Gründungsbekanntgabe der Soncino-Gesellschaft]

9) Jüdisch-liberale Zeitung: Organ der Vereinigung für das liberale Judentum e.V. (Berlin), Jg. 4, Nr. 20 (27.06.1924), 2. Beilage „Jüdische Bücher“ [Gründungsbekanntgabe, Nennung der Mitglieder des Ehrenausschusses]

1 Pressemappe der Soncino-Gesellschaft der Freunde des jüdischen Buches, Archiv des Jüdischen Museums Berlin (JMB), Sammlung Soncino-Gesellschaft, DOK 93/502/170-171. 
10) Jüdisch-liberale Zeitung: Organ der Vereinigung für das liberale Judentum e.V. (Berlin), Jg. 4, Nr. 21 (04.07.1924) [kurze Angabe der Geschäftsstelle der Soncino-Gesellschaft auf deren Bitte hin]

11) Jüdische Rundschau (Berlin), Jg. 29, Nr. 101 (19.12.1924), S. 731, Chanukka-Beilage „Aus dem Schauspiel ,Die Umkehr des Abtrünnigen“ von Arnold Zweig, 4. Akt, 4. Szene [Szene aus dem unveröffentlichten Drama „Der Abtrünnige“von Arnold Zweig, dessen ersten Teil die Soncino-Gesellschaft für ihre Mitglieder veröffentlichen wird]

12) Wiener Morgen-Zeitung (Wien), Jg. 7, Nr. 2117 (11.01.1925), S. 5/6, „Die Neubelebung der jüdischen Buchkunst. Die Soncino-Gesellschaft in Berlin“, Verfasser: o.r. [Bericht zur Entstehung und Arbeit der Soncino-Gesellschaft]

13) Jüdisch-liberale Zeitung: Organ der Vereinigung für das liberale Judentum e.V. (Berlin), Jg. 5, Nr. 13 (27.03.1925), Beilage „Soncino-Gesellschaft der Freunde des jüdischen Buches E.V. Berlin“ [Ziel der Gesellschaft und bisherige Veröffentlichung]

14) Jüdische Rundschau (Berlin), Jg. 30, Nr. 9 (30.01.1925), S. 81, „Soncino-Gesellschaft“, Verfasser: Dr. Kirschner [Besprechung der ersten Publikation]

15) Das jüdische Echo: Bayerische Blätter für die jüdischen Angelegenheiten; Mitteilungen der Zionistischen Vereine Bayerns (München), Jg. 12, Nr. 17 (24.04.1925), S. 314 [Veröffentlichung auf Bitte der Soncino-Gesellschaft: Ziel und erste Publikation der Soncino-Gesellschaft]

16) Jüdische Presszentrale Zürich und jüdisches Familienblatt für die Schweiz (Zürich), Jg. 8, Nr. 340 (17.04.1925), S. 13, „Soncino Gesellschaft der Freunde des Jüdischen Buches E.V. Berlin." [Text wie in Nr. 15]

17) Selbstwehr: jüdisches Volksblatt (Prag), Jg. 18, Nr. 26 (27.06.1924), S. 5, „Eine Gesellschaft der Freunde des jüdischen Buches“, Verfasser: Jüdische Telegraphen-Agentur GmbH (Berlin) [Gründungsbekanntgabe der Soncino-Gesellschaft]

18) Kölnische Zeitung (Köln), Abend-Ausgabe, Nr. 452A (28.06.1924), Beilage [Gründungsbekanntgabe der Soncino-Gesellschaft]

19) Neue Freie Presse (Wien), Abendblatt, Nr. 21506 (25.07.1924), S. 1, „Kleine Chronik“ [enthält einen Abschnitt über die Gründung der Soncino-Gesellschaft]

20) Das jüdische Echo: Bayerische Blätter für die jüdischen Angelegenheiten; Mitteilungen der Zionistischen Vereine Bayerns (München), XI, Nr. 27 (04.07.1924), S. 153, „Literarisches Echo" [Gründungsbekanntgabe der Soncino-Gesellschaft]

21) Leipziger Jüdische Zeitung: Wochenblatt für die gesamten Interessen des Judentums (Leipzig), Jg. 3, Nr. 26 (29.06.1924), S. 2, „,Freunde des jüdischen Buches““ [Gründungsbekanntgabe der Soncino-Gesellschaft]

22) Jüdische Presszentrale Zürich und jüdisches Familienblatt für die Schweiz (Zürich), Jg. 7, Nr. 300 (03.07.1924), S. 6, „Soncino-Gesellschaft der Freunde des jüdischen Buches“, Verfasser: Arnold Zweig [Gründungsbekanntgabe der Soncino-Gesellschaft]

23) Börsenblatt für den deutschen Buchhandel: Fachzeitschrift für Verlagswesen und Buchhandel (Leipzig), Nr. 170 (01.08.1924), S. 10114, „Neue bibliophile Gesellschaft“ [Gründungsbekanntgabe der Soncino-Gesellschaft]

24) keine Quellenangabe, kein Verfasser, kein Titel, in russischer Sprache [Gründungsbekanntgabe]

25) Jüdische Illustrierte Zeitung: יידישע אילוסטרירטע צייטונג (Berlin), Jg. 1, Nr. 7 (04.07.1924),

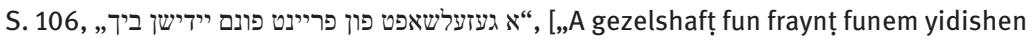
bikh“ = Eine Gesellschaft der Freunde des jüdischen Buches] [Gründungsbekanntgabe in jiddischer Sprache] 
26) Gutenberg-Museum (Bern), Jg. 10, Nr. 4 (Dezember 1924) [Anzeige für Werbeheft der Soncino-Gesellschaft vom Herbst 1924]

27) Kürschners deutscher Literatur-Kalender (Berlin [u.a.]), 1925, Nr. 42 [Anzeige mit zwei Feldern: 1) Gesellschaft, Ziel, Vorstand, Eintrittsgebühr 2) Jahresbeitrag, Veröffentlichungen, Versammlungen]

28) Mitteilungen der Jüdischen Reformgemeinde zu Berlin (Berlin), Jg. 8, Nr. 4 (01.07.1925), S. 2/3, „Von unserer Bibliothek“, Verfasser: Amtsgerichtsrat Walther Michaelis [Erläuterungen zum Einrichten einer Bibliothek, erwähnt in der letzten Spalte die SoncinoGesellschaft als Wegweiser für jüdische Tradition und deren Wiederbelebung]

29) Katalog / S. Martin Fraenkel (Berlin), Katalog Nr. 48 (März 1926) „Bibliophilendrucke. Agnon, S. J.“

30) Frankfurter Zeitung (Frankfurt a. M.), Literaturblatt (23.10.1925), S. 4 [Anzeige, SoncinoBlätter, Heft 1-2]

31) Börsenblatt für den deutschen Buchhandel: Fachzeitschrift für Verlagswesen und Buchhandel (Leipzig), Anzeige von Otto v. Holten, Berlin [Anzeige der Soncino-Blätter]

32) Börsenblatt für den deutschen Buchhandel: Fachzeitschrift für Verlagswesen und Buchhandel (Leipzig), Nr. 280 (01.12.1925), S. 19246 [Anzeige der Soncino-Blätter, genauere Inhaltsangabe Heft 1-2]

33) Auktion / Paul Graupe (Berlin), Auktion 53 (30. und 31.10.1925), 2 Angebote: No. 256 Der Prophet Jona, No. 1582 Soncino-Gesellschaft Sefer Meschalim

34) Katalog / Karl W. Hiersemann, Buchhändler und Antiquar (Leipzig), Katalogheft 559 (Januar 1926), Angebot No. 970 Sefer Meschalim

35) Der Orden Bne Briss: Mitteilungen d. Großloge für Deutschland VIII. U.O.B.B. (Berlin), (01.10.1925) „Das schöne jüdische Buch als Kulturfaktor“, Verfasser: Br. Dr. Siegfried Wolff (Timend. Jub.-Loge.) (Vorsitzender der Soncino-Gesellschaft der Freunde des jüdischen Buches) [Beschreibung der Soncino-Gesellschaft]

36) Orientalische Mitteilungen: aus dem Antiquariat Markert \& Petters (Leipzig) Jg. 1, (27.03.1926), Angebot No. 481 Soncino-Blätter

37) Kirjath sepher : bibliographical quarterly of the Jewish National and University Library Jerusalem (Jerusalem), Jg. 2, Nr. 4 (März 1926), S. 241, 2 Angebote: No. 504 SoncinoBlätter, No. 507 Bericht über die Tätigkeit der Soncino-Gesellschaft 1925 (auf Deutsch)

38) Jüdisch-liberale Zeitung: Organ der Vereinigung für das liberale Judentum e.V. (Berlin), Jg. 5, Nr. 50 (11.12.1925), 2. Beilage „Bücherschau“, Verfasser: Robert Hirschfeld, Berlin [Artikel über Soncino-Blätter und neue Veröffentlichungen]

39) Börsenblatt für den deutschen Buchhandel: Fachzeitschrift für Verlagswesen und Buchhandel (Leipzig), Nr. 3 (05.01.1926), S. 14, „Soncino-Blätter“, Verfasser J.G. [genaue Angaben/Besprechungen zu den Blättern]

40) Monatsschrift für Geschichte und Wissenschaft des Judentums (Frankfurt a. M.), Jg. 69, Neue Folge Jg. 33, Heft 7 (Dezember 1925), S. 457, Zeitschriftenschau (Auszüge) [Anzeige und Besprechung der Neuerscheinung der Soncino-Blätter]

41) Vertrauliche Mitteilungen des Deutschen Verlegervereins (Leipzig), Nr. 486 (April 1926), S. 12 „Neue Werbe-Anschriften der Adressenstelle“ [Anschriften der Mitglieder jetzt für 6 M lieferbar]

42) Börsenblatt für den deutschen Buchhandel: Fachzeitschrift für Verlagswesen und Buchhandel (Leipzig), (27.04.1926) [Anzeige für Soncino-Blätter, Heft 1]

43) Wiener Morgenzeitung (Wien), Jg.7, Nr. 2417 (14.11.1925), S. 5, „Jüdische Medailleure in Polen“ [Abdruck aus dem Artikel von Moritz Simon in den Soncino-Blättern] 
44) Wiener Morgenzeitung (Wien), Jg.7, Nr. 2418 (15.11.1925), S. 11, „Wiedererweckung des jüdischen Buches“ [zur Soncino-Gesellschaft und den Soncino-Blättern]

45) Jüdische Presszentrale Zürich und jüdisches Familienblatt für die Schweiz (Zürich), Jg. 8, Nr. 375 (18.12.1925), S. 14, „Das jüdische Buch als Gegenstand der Bibliophilie“ [Bericht über den gleichnamigen Vortrag von Hermann Meyer am 01.12.1925 beim Berliner Bibliophilen-Abend, wo er auch die Soncino-Gesellschaft erwähnte]

46) Berliner Tageblatt und Handels-Zeitung (Berlin), Jg. 55, Nr. 114 (09.03.1926) [zwei Todesanzeigen für Dr. jur. Siegfried Wolff von der Familie und dem Vorstand der SoncinoGesellschaft]

47) Jüdische Rundschau (Berlin), Jg. 31, Nr. 20 (12.03.1926), S. 150, Verfasser: b.k. [Nachruf auf Siegfried Wolff, erwähnt dessen Mitgliedschaft in der Soncino-Gesellschaft]

48) [Todesanzeige, ohne Quellenangabe, für Siegfried Wolff von der Familie]

49) Israelitisches Familienblatt (Hamburg), Jg. 28, Nr. 10 (11.03.1926), S. 3, „Todesfall“ [Nachricht vom Tod Siegfried Wolffs, erwähnt Soncino-Gesellschaft]

50) Zentralblatt für Bibliothekswesen (Leipzig), Jg. 43, (August 1926), S. 395-397, Verfasser: Gottschalk, Berlin [3 Publikationsseiten, Literaturberichte und Anzeigen, Besprechung der Soncino-Blätter]

51) Deutsche Literaturzeitung für Kritik der internationalen Wissenschaft (Berlin), Neue Folge, Jg. 3, Heft 16 (17.04.1926), Spalte 745-748, Abschnitt Allgemeinwissenschaftliches: Sammelwerke, Gelehrtenwesen, Schrift-, Buch- und Bibliothekskunde „Soncino-Blätter“, Verfasser: Max Joseph Husung, Berlin [Rezension]

52) Deutsche Literaturzeitung für Kritik der internationalen Wissenschaft (Berlin), Neue Folge, Jg. 2, Heft 40 (03.10.1925), Spalte 1937-1940, „Lederschnittbände des XIV. Jahrhunderts. Gesammelt und herausgegeben von Martin Bollert"Verfasser: Max Joseph Husung, Berlin [Rezension, Querverweis zu Husungs Aufsatz zum jüdischen Lederschnitt in den SoncinoBlättern]

53) Handbuch des Kunstmarktes. Kunstadressbuch für das deutsche Reich, Danzig und Deutsch-Österreich (Berlin), (1926) [Adresseintrag für Meyer, Hermann, in der Gruppe Sammler und Bibliophile in Berlin]

53a) Przewodnik bibliograficzny: miesięcznik dla wydawców, księgarzy, antykwarzy, jako też dla czytających i kupujących książki / Biblioteka Narodowa (Lwów) [Biographischer Führer: Monatszeitschrift für Verlage, Buchhändler, Antiquariate als auch für Leser und Käufer des Buches], Jg. 7, Heft 8 (1926) „Organisation Jüdischer Bücherfreunde“ [Original Polnisch, Text über die Soncino-Gesellschaft in der Pressemappe nur in deutscher Übersetzung]

54) Zentralblatt für Bibliothekswesen (Leipzig), Jg. 43, (Januar 1926), S. 30-32, Verfasser: Husung, Berlin [Rezension zu Martin Bollert: „Lederschnittbände des 15. Jahrhunderts“, erwähnt jüdischen Lederschnitt, aber nicht Soncino]

55) Der Schild: Zeitschrift des Reichsbundes jüdischer Frontsoldaten (Berlin), Jg. 5 (1926), S. 165, „Der Pavillon ,Hygiene der Juden“ auf der Düsseldorfer ,Gesolei““, Abschnitt „Die Veranstalter der Sondergruppe ,Hygiene der Juden““ [nur ein Teil des Artikels, erwähnt die Soncino-Gesellschaft als mitarbeitende Organisation]

56) Jüdisches Wochenblatt (Frankfurt a. M.), Jg. 3, Nr. 12 (19.03.1926), S. 108, „Ein PeßachGeschenk" [zur Peßach-Hagada des Gerschom Kohen im Neudruck von Soncino]

57) Zeitschrift für Bücherfreunde: Monatsheft für Bibliophilie und verwandte Interessen; Organ der Gesellschaft der Bibliophilen, der Deutschen Buchgewerbekünstler und der Wiener Bibliophilen-Gesellschaft (Leipzig), Verfasser: G.W. [Rezension zu Soncino-Blätter] 
58) Mitteilungen des Kartells Jüdischer Verbindungen (Berlin), Jg. 3, Nr. 11 (01.05.1924) [Anzeige zur Gründungsbekanntgabe]

59) Numismatisches Literaturblatt (Halle S.), Jg. 43 (Mai 1926), S. 2078f., „Moritz Simon: Juden als Medailleure in Polen“ [Anzeige zu den Soncino-Blättern] und „Samuel Raffaeli: Jewish Coinage and the date of the Bar-Kokhbah revolt“ [Anzeige, kein Verweis auf Soncino]

60) Zeitschrift für Bücherfreunde: Monatsheft für Bibliophilie und verwandte Interessen; Organ der Gesellschaft der Bibliophilen, der Deutschen Buchgewerbekünstler und der Wiener Bibliophilen-Gesellschaft (Leipzig), Jg. 18, (Mai/Juni 1926) [Anzeige zu „Suezkint der jude von trimberg: minnelieder“, Publikation der Soncino-Gesellschaft]

61) Jüdische Presszentrale Zürich und jüdisches Familienblatt für die Schweiz (Zürich), Jg. 9, Nr. 408 (20.08.1926) [Kurzbesprechung der Soncino-Blätter sowie Abdruck eines hebräischen Druckerzeichens]

62) Jüdische Rundschau (Berlin), Jg. 31, Nr. 72 (14.09.1926), S. 519, „Zur Notlage der hebräischen Literatur" Verfasser: Heinrich Loewe [Leserbrief in Reaktion auf den Artikel „Ueber die Notlage der hebräischen Literatur"vom 13.08.1926 (siehe Nr. 64), bezeichnet Soncino-Verlag als Teillösung des Problems; unter dem Leserbrief Hinweis auf den Tätigkeitsbericht der Soncino-Gesellschaft]

63) Chvila [Augenblick] (Lemberg), Nr. 2479 (11.02.1926) „Przjaciele książki żydowskiej“ [Freund des jüdischen Buches], Verfasser: Izak Lewin [in Polnisch, Fußnote erwähnt Soncino-Blätter]

64) Jüdische Rundschau (Berlin), Jg. 31, Nr. 63 (13.08.1926), S. 454/455, „Ueber die Notlage der hebräischen Literatur“, Verfasser: H.K. [Artikel zum Leserbrief in Nr. 62, in der Pressemappe falsch datiert]

65) Jüdische Presszentrale Zürich und jüdisches Familienblatt für die Schweiz (Zürich), Jg. 9, Nr. 416 (15.10.1926) „Druckerzeichen des Marco Antonio Giustaniani aus Israel Aschkenasi, B‘Sakim U-K‘Thawim, Venedig 1546 (zur Verfügung gestellt von der SoncinoGesellschaft)“ [nur Bild]

66) Literarische Mitteilungen, Nr. 2 der Buchhandlung Goldschmidt Gun?tt [unleserlich], Hamburg, Grindelallee 85 (20.10.1926) „Soncino“ [kurze Mitteilung zur SoncinoGesellschaft]

67) Berliner Morgenpost (Berlin), Jg. 28, (29.11.1926) [Ankündigung der Jahresversammlung der Soncino-Gesellschaft am 05.12.1926]

68) Jüdisches Wochenblatt (Frankfurt a. M.), Jg.3, Nr. 47 (03.12.1926), S. 420, Vereinskalender Berlin [Ankündigung der Jahresversammlung der Soncino-Gesellschaft am 05.12.1926]

69) Jüdisch-liberale Zeitung: Organ der Vereinigung für das liberale Judentum e.V. (Berlin), Jg. 6, Nr. 50 (10.12.1926), Beilage, „Aus den Gemeinden“ [Bericht über die Jahresversammlung der Soncino-Gesellschaft vom 4. und 5. 12.1926]

70) Israelitisches Familienblatt [(Hamburg), Jg. 28, (16.12.1926) „Jahresversammlung der Soncino-Gesellschaft" [Text wie Nr. 69]

71) The Reform advocate (Chicago), (23.10.1926), S. 379/380 Verfasser: Dr. M. Jung, [Kurzbesprechung von „Die Fabeln des Kuhbuches in Uebertragung. Mit einem Vorwort von Professor Dr. Aron Freiman. Soncino-Gesellschaft, Berlin C.2“, in Englisch]

72) B'nai B'rith Magazine (März 1926), S. 180/181, „The Prague Haggadah“, Verfasser: Samuel S. Cohen [Rezension zur Hagada von Gerschom Cohen, 2 Abbildungen]

73) פרייע שריפטן פארן יידישן סאציאליסטישן געדאנק 
[Fraye shrifțn farn yidishn sotsyalisțishn gedank = Freie Schriften von jüdisch sozialistischen Gedanken], (Warschau [u.a.]), (1927), Bd. 2 , 1926, שון נייע ביכער. שמואל לעווין, צ.א., צייטווע.,", Verfasser.

[Fun naye bikher. Shmuel Leṿin, „Zeyțyende“ Birlin 1926 = Von neuen Büchern. Schmuel Lewin. „Zeitwende“ Berlin 1926, Verfasser: A. V..] [in Jiddisch]

74) A) Menorah: jüdisches Familienblatt für Wissenschaft/Kunst und Literatur (Wien/Frankfurt a.M.), Jg. 5, Nr. 9 (September 1927), S. 561, Verfasser: N. [Rezension zu Samuel Lewin: Zeitwende]

B) Jevrejski Almanah 5688 (Vrsac [=Werschetz, Voijvodina, Serbien]), (1927), S. 172-174 [in Serbisch, Rezension zu Samuel Lewin: Zeitwende]

75) 1928 „Lukull vertreibt den Bücherteufel heute vom Soncino Turm“, Zeichnung von Werner Saul

76) Jüdische Presszentrale Zürich und jüdisches Familienblatt für die Schweiz (Zürich), Jg. 9, Nr. 426 (24.12.1926) „Druckerzeichen von Tobijah Foa“ [Druckerzeichen, von der SoncinoGesellschaft zur Verfügung gestellt]

77) Wiener Morgenzeitung (Wien), Literaturbeilage „Das Buch der Zeit“, Jg. 9, Nr. 2839 (23.01.1927), S. 9, Verfasser: S.S. [Rezension zu Samuel Lewin: Zeitwende]

78) Jüdische Rundschau (Berlin), Jg. 31, Nr. 95 (03.12.1926), S. 690, Vermischtes [Ankündigung der Jahresversammlung der Soncino-Gesellschaft am 05.12.1926]

79) Jüdische Rundschau (Berlin), Jg. 32, Nr. 16 (25.02.1927), S.113, Bücherschau, Verfasser: Eugen Hoeflich [Rezension zu Samuel Lewin: Zeitwende]

80) Vossische Zeitung: Berlinische Zeitung von Staats- und gelehrten Sachen (Berlin), Sonntagsausgabe, Nr. 204 (01.05.1927), Literarische Umschau: Beilage zur Vossischen Zeitung vom Sonntag, 1. Mai, Nr. 18, [S. 33] „Ein deutsch-polnischer Dichter. S. Lewins ,Zeitwende““, Verfasser: Etta Federn-Kohlhaas [Rezension]

81) Mitteilungen der Jüdischen Reformgemeinde zu Berlin (Berlin), Jg. 10, Nr. 1 (01.01.1927), S. 5-6, „Vom Buche und von Büchern“, Verfasser: Amtsgerichtsrat Walther Michaelis [Bibliophilie und die Soncino-Gesellschaft]

82) B'nai B'rith: Mesícnik velkolóze pro Ceskoslovenský Stát = B'nai B'rith: Monatsblätter d. Grossloge f. d. Cechoslovak. Staat [Prag], Jg. 6, (Februar 1927) [Ausschnitt eines Artikels zur Soncino-Gesellschaft]

83) Jüdisches Wochenblatt (Frankfurt a. M.), Jg. 5, Nr. 32 (17.08.1928), S. 263, Aus der Welt des Buches, Notizen [Mitteilung über den Versand des dritten Nachrichtenblattes der SoncinoGesellschaft]

84) Jüdisches Wochenblatt (Frankfurt a. M.), Jg. 5, Nr. 43 (09.11.1928), S. 362, Aus der Welt des Buches „Eine Monumentalausgabe der hebräischen Bibel“ [Mitteilung]

85) Wochenblatt für den Synagogenbezirk Halle a. S. (Halle, S.), Jg. 3 (19.10.1928) [Mitteilung über neue Druckschrift und Bibeldruckvorhaben der Soncino-Gesellschaft]

86) Deutsche israelitische Zeitung: Organ für die Gesamtinteressen des Judentums (Regensburg), Jg. 45, (06.12.1928), Verfasser: F.L. [Rezension des Romans „Zeitwende“von Samuel Lewin]

87) Jüdisch-liberale Zeitung: Organ der Vereinigung für das liberale Judentum e.V. (Berlin), Jg. 9, Nr. 6 (08.02.1929), Beilage, „Vereinsnachrichten“ [Ankündigung der Mitgliederversammlung am 17.02.1929 sowie über die Verteilung des Bibelprobedrucks]

88) Die Stimme: Jüdische Zeitung (Wien), Jg. 2, Nr. 59 (14.02.1929), S.10 [Mitteilung zum Erscheinen des Probedrucks der Bibel und zur anstehenden Jahresversammlung der Soncino-Gesellschaft] 
89) B‘nai B'rith: Mesícnik velkolóze pro Ceskoslovenský Stát = B‘nai B'rith: Monatsblätter d. Grossloge f. d. Cechoslovak. Staat [Prag], Jg. 6, Nr. 5 (Juni 1927), S. 295, „Neue SoncinoPublikationen“, Verfasser: t. [Mitteilung]

90) Die Stimme: Jüdische Zeitung (Wien), Jg. 1, Nr. 25 (21.06.1928), S. 12, „Soncino-Blätter“, Verfasser: ie [Mitteilung zum zweiten Jahresband]

91) Bayerische Israelitische Gemeindezeitung: Nachrichtenblatt der Israelitischen Kultusgemeinden in München, Augsburg, Bamberg und des Verbandes Bayerischer Israelitischer Gemeinden (München), Jg. 5, Nr. 4 (15.02.1929), S. 56, „Eine hebräische Monumentalbibel“ [Mitteilung zum Probedruck der Bibel sowie zur Mitgliederversammlung am 16./17.02.1929]

92) Berliner Adreßbuch: Eintrag für Soncino-Gesellschaft

93) Jüdische Rundschau (Berlin), Jg. 33, Nr. 46 (12.06.1928), S. 334 „,Soncino-Gesellschaft (Zweiter Jahrgang der Soncino-Blätter)“, Verfasser: Dr. B. Kirschner [Rezension]

94) Jüdische Wochenzeitung für Wiesbaden und Umgebung (Wiesbaden), Jg. 3, (15.02.1929) „Eine hebräische Monumentalbibel“ [Mitteilung zur Probedruckausgabe und Mitgliederversammlung]

95) Jüdisches Gemeindeblatt für Anhalt und Umgegend (Dessau), Jg. 4, Nr. 18 (15.02.1929) „Eine hebräische Monumentalbibel“ [Mitteilung zur Probedruckausgabe und Mitgliederversammlung]

96) Allgemeines Jüdisches Familienblatt: Wochenschrift für Politik, Literatur, Kunst und Wissenschaft (Leipzig), Jg. 10, (22.02.1929) „Jahresversammlung der SoncinoGesellschaft“" [Bericht über die Jahrestagung]

97) Jüdisches Jahrbuch. Berlin 1929 [Eintrag der Daten der Soncino-Gesellschaft und Zweck der Gesellschaft]

98) Sperlings Zeitschriften- und Zeitungsadressbuch: Handbuch der deutschen Presse. Leipzig 1930 [Eintrag zu Soncino-Blätter]

99) Redaktion der Minerva, WI 0, Genthiner Str. 38, Eintrag Nr. 4, Soncino-Gesellschaft der Freunde des Jüdischen Buches (E.V.)

100) Israelitisches Familienblatt [(Hamburg), Jg. 31, (02.03.1929) „Das jüdische Schulbuch. Ein Nachwort zur Tagung der Soncino-Gesellschaft“, Verfasser: Dr. A. Horodisch, Berlin

101) Berliner Tageblatt (06.04.1929), Verfasser: -n. [Mitteilung zu „Gelegentliches von Albert Einstein", Publikation der Soncino-Gesellschaft]

102) Jüdisches Gemeindeblatt für Anhalt und Umgegend (Dessau), Jg. 4 (01.03.1929) „SoncinoGesellschaft“ [Bericht über die Jahresversammlung am 17.02.1929]

103) Israelitisches Familienblatt [(Hamburg), Jg. 28, (21.02.1929) „Tagung der ,Freunde des jüdischen Buches ““, Verfasser: (Ita.) [Bericht über die Jahresversammlung am 17.02.1929]

104) Jüdische Presse: Organ für die Interessen des orthodoxen Judentums (Wien-Bratislava), Jg. 15, Nr. 8 (22.02.1929), S. 4-5, „Eine hebräische Monumentalbibel“, Verfasser: B. [Meldung über das Bibelprojekt und seinen Druck]

105) Jüdische Rundschau (Berlin), Jg. 34, Nr. 12 (12.02.1929), S. 76, Vermischtes [Mitteilung zur Probedruckausgabe der hebräischen Bibel und Mitgliederversammlung]

105a) Berliner Tageblatt und Handels-Zeitung (Berlin), Jg. 58 (22.03.1929) [Eintrag zu Ernst Simon: „Zum Problem des jüdischen Witzes“, Druck der Soncino-Gesellschaft]

106) Jüdisch-liberale Zeitung: Organ der Vereinigung für das liberale Judentum e.V. (Berlin), Jg. 9, Nr. 8 (22.02.1929), Beilage, „Aus den Gemeinden“ [Bericht über die Jahresversammlung vom 17.02.1929 und die dort gehaltenen Vorträge] 
107) Das jüdische Echo [Bayerische Blätter für die jüdischen Angelegenheiten; Mitteilungen der Zionistischen Vereine Bayerns (München), Jg. 16 (08.03.1929) [Bericht über die Jahresversammlung vom 17.02.1929]

108) Jüdische Rundschau (Berlin), Jg. 34, Nr. 15 (22.02.1929), S. 94, „Soncino-Gesellschaft“, Verfasser: B. St. (Berta Badt-Strauß) [Bericht über die Jahresversammlung vom 17.02.1929]

109) Selbstwehr: jüdisches Volksblatt (Prag), Jg. 23, (15.02.1929) „Tarbuth: Ein neues Kleid“, Verfasser: F.E. (Friedrich Eckstein) [zum Bibeldruckvorhaben der Soncino-Gesellschaft]

110) Gemeindeblatt der Israelitischen Religionsgemeinde zu Leipzig: amtliches Nachrichtenblatt der Gemeindeverwaltung (Leipzig), Jg. 5, Nr. 12 (22.03.1929), „Neue Bücher. Hebräische Bibel der Soncino-Gesellschaft“ [Bericht über die neue Drucktype]

111) Jüdische Presszentrale Zürich und jüdisches Familienblatt für die Schweiz (Zürich), Jg. 12, Nr. 542 (19.04.1929), S. 16, „Gelegentliches zu Albert Einstein“ [Mitteilung]

112) Frankfurter Zeitung (Frankfurt am Main), (April 1929), Verfasser: E.B. (Ernst Blau) [Mitteilung zur 9. Publikation „Jesus Sirach. Mit Holzschnitten von Steinhardt"]

113) Jüdisches Wochenblatt für Magdeburg und Umgegend (Magdeburg), Jg. 4, Nr. 12 (22.03.1929) „Zur Popularisierung der Bibel“ [Mitteilung zur Probedruckausgabe der Soncino-Bibel]

114) Nasz Przegląd (Warszawa), niedziela 27 (września 1931) „Wśród ksiąg jubileuszowych i pamiątkowych. Franz Rosenczweig“ [Unsere Besprechung (Warschau), Sonntag 27 (September 1931) „Unter den Jubiläums- und Gedenkbüchern. Franz Rosenzweig“] Verfasser: Prof. Dr. Majer Bałaban [in Polnisch, zu „Franz Rosenzweig. Ein Buch des Gedenkens “, Elfte Publikation der Soncino-Gesellschaft, Berlin 1930.]

115) Gemeindeblatt der Deutsch-Israelitischen Gemeinde zu Hamburg (Hamburg), Jg. 6, Nummer 11 (10.11.1930), S.1, „Franz Rosenzweig. Einem Großen zum Gedächtnis“, Verfasser: N. M. Nathan (Nathan Max Nathan) [Nachruf anlässlich des Begräbnisses, Vorschlag einer Gedächtnisstiftung, erwähnt das Buch des Gedenkens der SoncinoGesellschaft in einer Fußnote]

116) B'nai B’rith: Mesícnik velkolóze pro Ceskoslovenský Stát = B'nai B'rith: Monatsblätter d. Grossloge f. d. Cechoslovak. Staat [Prag], Jg. 7, Nr. 8 (1928), S. 365, Auszug aus den Verhandlungen der Tagung des Generalkomités der Grossloge für den tschechoslovakischen Staat J.O.B.B. am 28.09.1928 [Ausschnitt, Diskussion, ob Logen Mitglieder der Soncino-Gesellschaft werden sollen; Großloge beschließt, Mitglied zu werden]

117) [Mitteilung zum Versand der Nr. 3 des Nachrichtenblattes, ohne Quellenangabe]

118) B’nai B’rith: Mesícnik velkolóze pro Ceskoslovenský Stát = B'nai B'rith: Monatsblätter d. Grossloge f. d. Cechoslovak. Staat [Prag], Jg. 7, Nr. 8 (1928), S. 379-380 „Die SoncinoGesellschaft“, Verfasser: ft. (Dr. Friedrich Thieberger) [Artikel aus Anlass der Empfehlung an die Logen, der Soncino-Gesellschaft beizutreten]

119) Kb-Blätter Berlin, Jg. 19, Heft 3 (September/Oktober 1929), S. 60, „Mendelssohn - Feier der Soncino-Gesellschaft“, Verfasser: R.H. [Bericht über die Feier]

120) Journal of the Society of Oriental Research (Toronto), Jg. 13 (Juli 1929), Verfasser: John A. Maynard [in Englisch, Kurzrezension zu Soncino-Blätter Band 11]

121) Berliner Tageblatt und Handels-Zeitung (Berlin), Jg. 59, (26.01.1930) „Luther im Jargon“, Verfasser: J.K. [Rezension zum Aufsatz „Zur Geschichte der hebräischen Druckereien in Polen" von Prof. Dr. Majer Balaban in den Soncino-Blättern]

122) Central-Verein-Zeitung: Blätter für Deutschtum und Judentum; Organ des Central-Vereins Deutscher Staatsbürger jüdischen Glaubens e.V. (Berlin), Jg. 9, Nr. 7 (14.02.1930), S. 80, 
„Freunde des jüdischen Buches. Das Werk der Soncino-Gesellschaft“, Verfasser: Dr. Eugen Pinner

123) Prager Presse (Prag), Jg. 9, Nr. 32 (11.08.1929) „Bibliophiles Kabinett. Die Berliner Soncino-Gesellschaft und ihre Publikationen“, Verfasser: O. Donath

124) Central-Verein-Zeitung: Blätter für Deutschtum und Judentum; Organ des Central-Vereins Deutscher Staatsbürger jüdischen Glaubens e.V. (Berlin), Jg. 9, Nr. 15 (11.04.1930), S. 197 „Das Stiftungsfest der Soncino-Gesellschaft“, Verfasser: P. [Bericht über das Fest am 29.03.1930]

125) Vossische Zeitung: Berlinische Zeitung von Staats- und gelehrten Sachen (Berlin), Abendausgabe, Nr. 471 (05.10.1927), [S. 2] „Fedor v. Zobeltitz’ 70. Geburtstag““ [SoncinoGesellschaft wird als Gratulant erwähnt]

126) Philobiblon: eine Zeitschrift für Bücherliebhaber (Leipzig [u.a.]), Jg. 2, (1929), S. 132, „Soncino-Gesellschaft“, Verfasser: H.M. [Bericht zur 3. Jahresversammlung am 20.05.1929]

127) Jüdisch-liberale Zeitung: Organ der Vereinigung für das liberale Judentum e.V. (Berlin), Jg. 10, Nr. 37 (10.09.1930), Beilage, „Aus den Gemeinden“ [Mitteilung zum 50.Geburtstag des Amtsgerichtsrats Walther Michaelis am 12.09.1930, erwähnt dessen Interesse an der Soncino-Gesellschaft]

128) The New York Times Book Review (New York, NY), Jg. 36, (23.08.1931), S. 2, „Fine Books From the Presses of Germany. The Bibliophile of That Country Can Choose From a Rich Diversity of Printing, Decorating and Binding“, Verfasser: Annemarie Meiner, Munich [in Englisch, erwähnt Soncino]

129) ליטערארישע בלעטער אילוסטרירטע וואכנשריפט פאר ליטעראטור טעאטער און קונסט. [Lițerarishe Blețer: Ilusțrirțe Ỵokhenshrifț far Lițerațur, Ṭeater un Ḳunsț = Literarische Blätter: Illustrierte Wochenschrift für Literatur, Theater und Kunst] (Warschau), Jg. 4, Nr. 11 (18.03.1927), Verfasser: מ. ווייסבירג [M. Ỵaysbirg= Weißberg] [in Jiddisch]

130) Keine Quellenangaben, ביבליאפיליע ביי יידו, [Bibliophilie bey Yido = Bibliophilie unter Juden) [in Jiddisch, erwähnt Soncino-Blätter im Text]

\section{Artikel, die in der Pressemappe nicht nummeriert sind}

The United Synagogue Recorder (New York/Newark, NJ), Jg. 8, Nr. 4 (Oktober 1928), S. 9, „Doctor H. Brody's Contribution to the Study of Medieval Hebrew Poetry. A Tribute on his $60^{\text {th }}$ Birthday“, Verfasser: Prof. Israel Davidson [unvollständig]

Selbstwehr: jüdisches Volksblatt (Prag), Jg. 22, (25.5.1928), S. 4 [Mitteilung zum 60. Geburtstag von Heinrich Brody]

Selbstwehr: jüdisches Volksblatt (Prag), Jg. 22 (25.5.1928) „Oberrabbiner Dr. Heinrich Brody und der Misrachi“, Verfasser: Dr. Oskar Wolfsberg, Berlin

Mitteilungsblatt des Landesverbandes Israelitischer Religionsgemeinden Hessens (Mainz), Jg. 5, (05.12.1930), S. 13, Verfasser: Dr. L. [Mitteilung zur Festschrift für Heinrich Brody]

[Der Orden Bne Briss: Mitteilungen der Großloge für Deutschland VIII. U.O.B.B (Berlin)], Jg. 10, Nr. 7 (September 1931), S. 345/355 „Bücher der Soncino-Gesellschaft“, Verfasser: ft. (F. Thieberger) [über die Neudrucke der Soncino-Gesellschaft]

Gemeindeblatt der Israelitischen Religionsgemeinde zu Leipzig: amtliches Nachrichtenblatt der Gemeindeverwaltung (Leipzig), Jg. 7, Nr.1 (02.01.1931), S. 7, „Neue Veröffentlichungen der Soncino-Gesellschaft der Freunde des jüdischen Buches“ 
Der Orden Bne Briss : Mitteilungen der Großloge für Deutschland VIII. U.O.B.B (Berlin), (Dezember 1930) [Mitteilung zum Eingang von vier Publikationen der SoncinoGesellschaft]

The Jewish Guardian (London), Jg. 12, Nr. 583 (28.11.1930), S. 4, „A Jubilee Volume“, Verfasser: Rabbi Dr. A. Marmorstein [in Englisch, Laudatio zum Geburtstag von Heinrich Brody] Der Israelit: ein Centralorgan für das orthodoxe Judentum (Frankfurt a. M.), Jg. 72, Nr. 31 (30.07.1931), S. 11, „Personalien. Zum 60. Geburtstag von Prof. Dr. Aron Freimann, Frankfurt a. M.“, Verfasser: Prof. Dr. Isaak Markon, Hamburg [erwähnt die von der SoncinoGesellschaft herausgegebene Festschrift für Aron Freimann]

Jüdische Wochenzeitung für Nassau (Wiesbaden), Jg. 5, Nr. 32 (07.08.1931), „Professor Aron Freimann 60 Jahre“

Jüdische Wochenzeitung für Nassau (Wiesbaden), Jg. 6, Nr. 8 (19.02.1932), „Eine Ortsgrupe

[sic!] Frankfurt der Soncino-Gesellschaft“

Jüdisches Gemeindeblatt: Mitteilungsblatt der Israelitischen Gemeinde; amtl. Organ der Gemeindeverwaltung Bremen (Bremen) ODER Jüdisches Gemeindeblatt: Mitteilungsblatt d. Israelitischen Gemeinde Bremen (Kassel), Jg. 4, Nr. 4 (24.02.1932) [Werbung für die Mitgliedschaft in der Soncino-Gesellschaft]

„Prof. Dr. Aron Freimann 60 Jahre“ [Artikel ohne Quellenangabe]

\section{Lose in der Pressemappe liegende Zeitungsauschnitte}

Nasz Przeglad (Warszawa), niedziela 16 (września 1931) „Wśród ksiąg jubileuszowych i pamiątkowych. III. Dr. Heinrich Brody“ [Unsere Besprechung (Warschau), Sonntag 16 (September 1931) „Unter den Jubiläums- und Gedenkbüchern. III. Dr. Heinrich Brody] Verfasser: Prof. Dr. Majer Bataban [in Polnisch, Festschrift für Heinrich Brody]

Bayerische Israelitische Gemeindezeitung: Nachrichtenblatt der Israelitischen Kultusgemeinden in München, Augsburg, Bamberg und des Verbandes Bayerischer Israelitischer Gemeinden (München), Jg. 6, Nr. 20 (15.10.1930), S. 316/317, „Bemerkungen über neuere jüdische Bücher und Zeitschriften“, Verfasser: Ludwig Feuchtwanger (in drei Abschnitten, Abschnitt 1 ,1. Neue Veröffentlichungen der Soncino-Gesellschaft der Freunde des jüdischen Buches")

Frankfurter Zeitung (Frankfurt a. M.), Lokalteil „Stadtblatt“ „Die Bibliophilen bei der SoncinoGesellschaft“, Verfasser: E.B. [Artikel zur Jahresversammlung, erwähnt Redner Horodisch]

„Die jüdische Buchdruckerei in Dyhernfurth“ [ohne Quellenangabe, Nachtrag zu einem Artikel in Heft 6 der Mitteilungen der Soncino-Gesellschaft]

B.Z. am Mittag (Berlin), Nr. 276 (26.11.1931), 1. Beiblatt, S. 4, „Die Berliner Museen stellen aus. Maria Slavona im Kronprinzenpalais“, Verfasser: Lothar Briegen [erwähnt Officina Serpentis]

Der Israelit: ein Centralorgan für das orthodoxe Judentum (Frankfurt a. M.), Jg. 71, Nr. 45 (06.11.1930), S. 12 [Mitteilung zur Veröffentlichung der Festschrift für Brody]

Vrijdagen (Amsterdam), Jg. 5, Deel 9, Nr. 8 (25.05.1928), S. 123, Verfasser: Dr. Simon Adler, Prag [in Holländisch, Artikel zu Heinrich Brody]

Mitteilungen der Jüdischen Reformgemeinde zu Berlin (Berlin), Jg. 17, Nr. 3 (15.03.1934), S. 1, „Die Soncino-Thora“, Verfasser: Walther Michaelis 
Gemeindeblatt der Jüdischen Gemeinde zu Berlin (Berlin), Jg. 24, Nr. $33 / 34$ (08.09.1934), S. 11, Spalte a „Wie das jüdische Buch entsteht. Zum 10jährigen Bestehen der SoncinoGesellschaft“, Photos und Montage: Erich Pincus [Bildercollage]

3 Zeitungsausschnitte auf Hebräisch, einer davon aus der Zeitung הצופה, S. 5f. [Artikel zu Chaim Brody, ohne Quellenangabe, Anfang fehlt]

\section{Pressemappe „Tagung der Soncino-Gesellschaft in Frankfurt a. Main am 24., 25. \& 26. Mai 1931“"}

„Jahresversammlung 1931 in Frankfurt a. M.“ In: Mitteilungen der Soncino-Gesellschaft. Nr. 7-10. März 1931. [Einladung zur Jahresversammlung wie in den Mitteilungen abgedruckt] „Soncino-Gesellschaft der Freunde des jüdischen Buches e.V.“ [Gedrucktes Heftchen mit Einladung zur Tagung in Frankfurt am Main vom 24. bis 26. Mai 1931, S. 1 Titelblatt, S. 2 leer, S. 3 „Programm der sechsten Jahresversammlung der Soncino-Gesellschaft“, S. 4-7 Programm]

Israelitisches Familienblatt (Hamburg), Jg. 33, Nr. 14 (01.04.1931), Frankfurter Ausgabe „Versammlung der Soncino-Gesellschaft“ [kurzer Bericht über die Versammlung in Frankfurt vom 26. März 1931, mit Vortrag von Herrmann Meyer über seine MendelssohnSammlung, danach Beratung über die kommende Jahresversammlung im Mai]

Der Israelit: ein Centralorgan für das orthodoxe Judentum (Frankfurt a. M.), Jg. 72, Nr. 14 (01.04.1931), S. 9, „Soncino-Gesellschaft“

Der Israelit: ein Centralorgan für das orthodoxe Judentum (Frankfurt a. M.), Jg. 72, Nr. 21 (21.05.1931), S. 8, „Der Soncino-Gesellschaft zum Gruß“, Verfasser: H.E. (Heinrich Eisemann) [Bericht zur Jahrestagung mit kurzem Abriss zur Geschichte der Bewegung „zu Gunsten des ,schönen Buches"]

Wormser Zeitung (Mainz), (26.05.1931) „,üdische Wissenschaftler besuchen Worms“, Verfasser: Adolf Tschirner [Kurzbericht über den Besuch der Tagungsgäste (ca. 40 Mitglieder) in Worms, Synagogenführung mit Rabbiner Dr. Holzer zu Kunstschätzen, Dombesichtigung mit A. Tschirner]

Der Israelit: ein Centralorgan für das orthodoxe Judentum (Frankfurt a. M.), Jg. 72, Nr. 22 (28.05.1931), S. 7, „Die Tagung der Soncino-Gesellschaft“ [Kurzbericht über die stattgefundenen Programmpunkte und Einladung zur Ausstellungsbesichtigung (1.-30. Juni)]

Frankfurter Zeitung (Frankfurt am Main), 2. Morgenblatt, Nr. 395 (30.05.1931), S. 2 „)Jahresversammlung der Soncino-Gesellschaft“, Verfasser: Edwin Baer [Bericht über die Tagung, Erwähnung der Gründung einer Ortsgruppe für Frankfurt und weitere Umgebung] Israelitisches Familienblatt (Hamburg), Jg. 33, Nr. 23 (04.06.1931) „Die Tagung der jüdischen Bücherfreunde. Jahresversammlung der Soncino-Gesellschaft (Sonderbericht für das ,Israelitische Familienblatt')“, Verfasser : Frl. Susmann [ausführlicher Bericht über die Programmpunkte der Tagung, kurze Wiedergabe der Vortragsinhalte]

Israelitisches Familienblatt (Hamburg), Jg. 33, Nr. 23 (04.06.1931) „Die Ausstellung ,Das schöne hebräische Buch der Frühzeit““, Verfasser Richard Ettinghausen [Bericht über die Bücher der Ausstellung und deren Besonderheiten / Geschichte]

Der Israelit: ein Centralorgan für das orthodoxe Judentum (Frankfurt a. M.), Jg. 72, Nr. 23 (04.06.1931), S. 9, „Soncino-Gesellschaft“, Verfasser: H.E. (Heinrich Eisemann) [Bericht zu den einzelnen Programmpunkten der Jahresversammlung] 
Frankfurter Zeitung (Frankfurt am Main), Abendblatt, Nr. 412 (05.06.1931), S. 2, „Bücher und Schriftrollen“, Verfasser: Dr. H. Gundersteiner [Bericht zur Ausstellung „Das illustrierte hebräische Buch bis zum Ende des 17. Jahrhunderts “ in der Frankfurter Stadtbibliothek. Die Ausstellung wurde im Anschluss an die Jahrestagung der Soncino-Gesellschaft eröffnet]

Central-Verein-Zeitung: Blätter für Deutschtum und Judentum; Organ des Central-Vereins Deutscher Staatsbürger jüdischen Glaubens e.V. (Berlin), Jg.10, Nr. 23 (05.06.1931), S. 293, „Soncino-Gesellschaft in Frankfurt a. M. 24. bis 26.Mai d.J.“, Verfasser: Dr. Felix Kauffmann, Frankfurt a. M. [Kurzbericht zu den Programmpunkten, besonders erwähnt die Austeilung von elf Festgaben, neun davon mit Titel und Stifter der Festgabe aufgezählt] Gemeinde-Zeitung für die israelitischen Gemeinden Württembergs (Stuttgart), Jg. 7, Nr. 9 (01.08.1931) „Im Dienste des jüd. Buches“ [Bericht über die Programmpunkte der Tagung]

Börsenblatt für den deutschen Buchhandel: bbb; Fachzeitschr. für Verlagswesen und Buchhandel (Leipzig), Nr. 142 (23.06.1931) „Ausstellung der Frankfurter Stadtbibliothek“, Verfasser: Alfred Schmidt, Wiesbaden [Beschreibung des Aufbaus der Ausstellung „Das illustrierte hebräische Buch bis zum Ende des 17. Jahrhunderts“ und einiger Werke] Jüdische Rundschau (Berlin), Jg. 36, Nr. 44 (09.06.1931), S. 273, Tagungen und Vorträge (Auszug aus dem JTADienst) [ohne Titel, Kurzbericht zur Jahrestagung]

Börsenblatt für den deutschen Buchhandel: bbb; Fachzeitschr. für Verlagswesen und Buchhandel (Leipzig), Nr. 140 (20.06.1931), Verfasser: Dr. Felix Kauffmann [ohne Titel]

Gemeindeblatt der Israelitischen Religionsgemeinde zu Leipzig: amtliches Nachrichtenblatt der Gemeindeverwaltung (Leipzig), Jg. 7, Nr. 24 (12.06.1931), S. 4, „Jahresversammlung der Soncino-Gesellschaft"

Jüdische Telegraphen-Agentur GmbH (Berlin), Jg. 10, Nr. 123 (03.06.1931), S. 4, „Im Dienste des juedischen Buches. Jahresversammlung der Soncino-Gesellschaft!“, Verfasser: H. M.

Jüdische Telegraphen-Agentur GmbH (Berlin), Jg. 10, Nr. 108 (13.05.1931), S. 6, „In den Pfingsttagen Jahresversammlung der Soncino-Gesellschaft in Frankfurt a. Main" [Ausblick auf das Tagungsprogramm]

Frankfurter Israelitisches Gemeindeblatt: amtliches Organ der Israelitischen Gemeinde (Frankfurt am Main), Jg. 9, Nr. 11 (Juli 1931), S. 350-351, „Hebraica-Ausstellung“, Verfasser: R. Ettinghausen [Bericht zur Führung durch die Ausstellung und Beschreibung einiger Objekte]

Jüdische Wochenzeitung für Nassau, (Wiesbaden), Jg. 5, Nr. 25, S.6 (19.06.1931), „Das illustrierte hebräische Buch bis 1600. Ausstellung der Frankfurter Stadtbibliothek vom 1.-21. Juni“, Verfasser: Alfred Schmidt, Wiesbaden [Beschreibung einiger ausgestellter Werke]

Gemeindeblatt der Israelitischen Religionsgemeinde zu Leipzig: amtliches Nachrichtenblatt der Gemeindeverwaltung (Leipzig), Jg. 7, Nr. 20 (15.05.1931), ,Jahresversammlung der Soncino-Gesellschaft" [Ankündigung der Tagung mit kurzer Programmbeschreibung] Jüdische Rundschau (Berlin), Jg. 36, Nr. 37 (15.05.1931), S. 232, Vermischtes [ohne Titel] Jüdisch-liberale Zeitung: Organ der Vereinigung für das liberale Judentum e.V. (Berlin), Jg. 11, Nr. 24-25 (24.06.1931), 2. Beilage, „Im Dienste des jüdischen Buches“ [Bericht über die Tagung der Soncino-Gesellschaft in Frankfurt]

Bayerische Israelitische Gemeindezeitung: Nachrichtenblatt der Israelitischen Kultusgemeinden in München, Augsburg, Bamberg und des Verbandes Bayerischer Israelitischer Gemeinden (München), Jg. 7, Nr. 12 (15.06.1931), S. 177-178, „Die Pfingsttagung der Akademie für die Wissenschaft des Judentums und der Soncino-Gesellschaft“ " $d$ der 
Ausschnitt im Pressespiegel enthält lediglich Abschnitt 2 „2. Die Tagung der SoncinoGesellschaft"]

Philobiblon: eine Zeitschrift für Bücherliebhaber (Leipzig [u.a.]), Jg. 4, Nr. 7 (1931), S.

291, „Soncino-Gesellschaft der Freunde des jüdischen Buches, Berlin“, Verfasser: K. [Kurzbericht zur Tagung, Nennung von fünf Festgaben] 